\title{
Fingerprint Based Authentication System using
} ARM7

\author{
Ambavarapu Bhavana ${ }^{1}$, M. Jasmine ${ }^{2}$ \\ ${ }^{1}$ M.Tech, PG Scholar, Department of ECE, Sri Venkatesa Perumal College of Engineering and Technology, Puttur -India \\ ${ }^{2}$ Associate Professor, Department of ECE, Sri Venkatesa Perumal College of Engineering and Technology, Puttur -India
}

\begin{abstract}
This project describes two state-of-the-arts and portable finger print based authentication and GSM based authentication system. While the authentication system allows secure access to a media center at home/banks/office/industries etc. The present scenario to operate a bank locker is with locks which are having keys. By this we can't say that we are going to provide good security to our homes/banks/offices etc. To provide perfect security and to make our work easier, we are taking the help of two different technologies viz. Embedded Systems and Biometrics. The main purpose of this project is to design and implement a high security system based on FINGERPRINT, PASSWORD and GSM technology which can be organized in bank, secured offices and homes.
\end{abstract}

Keywords: Fingerprint module, GSM, ARM7, Keypad.

\section{Introduction}

The proposed system will be developed on an authentication system using finger print and GSM technology. Biometrics deals with automated method of identifying a person or verifying the identity of a person based on the physiological or behavioral characteristic, and so are used for authentication in many of the online transactions. The biometric that has been chosen for implementation is fingerprint, since fingerprint biometric is easily available and highly reliable compared to many other biometrics. Finger print of the users is stored in first and the verified at the time of use. If fingerprint is matched to the trained prints then access was accepted again through GSM OTP was generated automatically and then send to registered mobile number. If it was typed by keypad then LCD displays authorized person accessed.

Fingerprint verification is one of the most reliable personal identification methods in biometrics. This system consists of microcontroller, GSM modem, Fingerprint scanner keyboard, and LCD. In this system fingerprint is matched then microcontroller sends the password to the authenticated person mobile number then the authenticated person enters the both passwords in the keyboard which was already given by the user and received from the microcontroller. if these two passwords are matched then the locker will be opened otherwise the microcontroller sends the warning message to the authenticated person mobile number and it will be remain in locked position, This system is more secure than other systems because two passwords required for verification.

\subsection{Existing System}

An automated teller machine (ATM) or cash machine is an electronic device that allows a bank's customers to make cash withdrawals and check their account balances without the need or human teller. Many ATMs also allow people to deposit cash or cheques, transfer money between their bank ac- counts, top up their mobile phones prepaid or even buy post- age stamps. In most modern ATMs, the customer identifies him or herself by inserting a plastic card with magnetic strip or plastic smart card with a chip that contains his or her account number. The customer then verifies his or her identity by entering a pass code (i.e.) personal identification number (PIN) of four digits. If the number is entered incorrectly several times consecutively (usually three), most ATMs will retain the card as a security precaution to prevent an unauthorized user form discovering the PIN by guesswork and so on. Moreover there is a limitation in transaction for the other bank customers in using the ATM of some other bank crossing the limit they have to pay transaction fees.

\subsection{Proposed System}

The proposed system will be developed on an authentication system using finger print and GSM technology. Biometrics deals with automated method of identifying a person or verifying the identity of a person based on the physiological or behavioral characteristic, and so are used for authentication in many of the online transactions. The biometric that has been chosen for implementation is fingerprint, since fingerprint biometric is easily available and highly reliable compared to many other biometrics. Finger prints of the users are stored in first and the verified at the time of use. If fingerprint is matched to the trained prints then access was accepted again through GSM OTP was generated automatically and then send to registered mobile number. If it was typed by keypad then LCD displays authorized person accessed.

\subsection{Scope}

The scope of study which is needed for the completion of this project involves the following criteria:

1)Architecture of LPC2148 knowledge

2)ARM7 programming in $C$ language.

3) The study of modem functions which involves AT commands.

4)The circuitry and devices that is needed to construct the devices and establish the necessary communication 


\section{International Journal of Science and Research (IJSR) \\ ISSN (Online): 2319-7064}

Index Copernicus Value (2013): 6.14 | Impact Factor (2015): 6.391

between the devices.

5)The study of GSM module along with its commands.

6) The communication between GSM modem, Finger print module and the microcontroller will be taken by the serial communication.

\section{Hardware Design of Proposed System}

The main aim of this project is to provide the security to your bank locker or for your home. This is the multi way security. To do this we are using fingerprint module, GSM module and keypad.

\subsection{Proposed system Design}

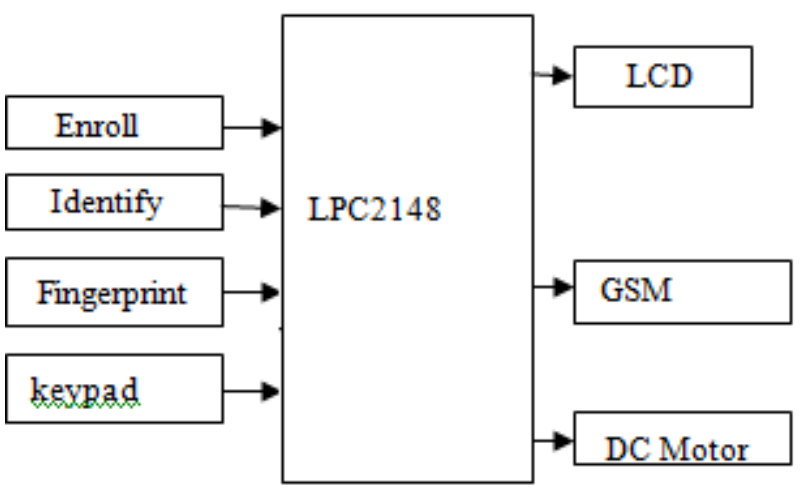

Figure 1: Block diagram of the proposed system

First of all the owner of the home or locker must register his fingerprint using fingerprint module. We can also register all of our family member's fingerprints also if we wish.

While at the time of opening the locker, then the authorized person, who's registered his fingerprint earlier, should give his fingerprint for checking. If it already a registered fingerprint, then only it will go further step. If not it will simply display that it is not registered and the locker will not be opened and also an alert message will be sent to the authorized owner of the locker.

If authorized person's fingerprint was matched, then the system will generate a One Time Password (OTP) to the owner's pre-registered mobile number. Then the owner should enter that one time password from the keypad provided at the locker side. If he enters the correct password then the locker will be opened and closed again after some delay. If he enter the wrong password then also an alert message "somebody was trying open your locker" to the locker owner. By this way we can provide the security to the bank lockers and houses also.

The code was written in embedded $\mathrm{C}$ language and compiled using Kiel micro vision 4 compiler. The generated hex file was dumped into the LPC2148 microcontroller using flash magic software through the UART port 0 .

(A) ARM7 (LPC 2148)

The LPC2148 microcontrollers are based on a 16-bit/32-bit ARM7TDMI-S CPU with real-time emulation and embedded trace support, that combine microcontroller with embedded high speed flash memory ranging from $32 \mathrm{kB}$ to $512 \mathrm{kB}$. A 128-bit wide memory interface and unique accelerator architecture enable 32-bit code execution at the maximum clock rate. For critical code size applications, the alternative 16-bit Thumb mode reduces code by more than $30 \%$ with minimal performance penalty.

\section{(B) FINGERPRINT}

This is a fingerprint sensor module with TTL UART interface for direct connections to microcontroller UART or to PC through MAX232 / USB-Serial adapter. The user can store the finger print data in the module and can configure it in 1:1 or 1: $\mathrm{N}$ mode for identifying the person. The FP module can directly interface with 3.3 or $5 \mathrm{v}$ Microcontroller. A level converter (like MAX232) is required for interfacing with PC serial port.

(C) GSM

GSM (Global System for Mobile communications) is an open, digital cellular technology used for transmitting mobile voice and data services. GSM (Global System for Mobile communication) is a digital mobile telephone system that is widely used in Europe and other parts of the world.

GSM uses a variation of Time Division Multiple Access (TDMA) and is the most widely used of the three digital wireless telephone technologies (TDMA, GSM, and CDMA). GSM digitizes and compresses data, then sends it down a channel with two other streams of user data, each in its own time slot. It operates at either the $900 \mathrm{MHz}$ or $1,800 \mathrm{MHz}$ frequency band. It supports voice calls and data transfer speeds of up to $9.6 \mathrm{kbit} / \mathrm{s}$, together with the transmission of SMS (Short Message Service).

\section{(D) KEYPAD}

This discussion shows how to connect a $4 \times 3$ keypad to a microcontroller, using just 4 lines. A keypad can be used for a number of applications.

- Dialling telephone numbers.

- Dialling a code for an alarm or door-entry.

\section{(E) DC Motors}

Electric motors are used to efficiently convert electrical energy into mechanical energy. Magnetism is the basis of their principles of operation. They use permanent magnets, electromagnets, and exploit the magnetic properties of materials in order to create these amazing machines.

\section{Software Design of Proposed System}

Firmware implementation deals in programming the microcontroller so that it can control the operation of the IC's used in the implementation. In the present work, we have used the Orcad design software for PCB circuit design, the Kiel $\mu \mathrm{v} 4$ software development tool to write and compile the source code, which has been written in the $\mathrm{C}$ language. The Flash magic programmer has been used to write this compile code into the microcontroller. 


\section{International Journal of Science and Research (IJSR) \\ ISSN (Online): 2319-7064}

Index Copernicus Value (2013): 6.14 | Impact Factor (2015): 6.391

Software Tools Required

- Orcad

- Kiel $\mu$ Vision4

- Flash Magic

Orcad is used for drawing the schematic diagram, it is mentioned above. Keiluv4, Flash magic are the two software tools used to program microcontroller. The working of each software tool is explained below in detail.

\subsection{Programming code description}

A compiler for a high level language helps to reduce production time. To program the LPC2148 microcontroller the Kiel $\mu \mathrm{v} 4$ is used. The programming is done in the embedded $\mathrm{C}$ language or Assembly language. Kiel $\mu \mathrm{v} 4$ is a suite of executable, open source software development tools for the microcontrollers hosted on the Windows platform.

One of the difficulties of programming microcontrollers is the limited amount of resources the programmer has to deal with. In personal computers resources such as RAM and processing speed are basically limitless when compared to microcontrollers. In contrast, the code on microcontrollers should be as low on resources as possible.

\subsection{Kiel Compiler}

Kiel compiler is software used where the machine language code is written and compiled. After compilation, the machine source code is converted into hex code which is to be dumped into the microcontroller for further processing. Kiel compiler also supports $\mathrm{C}$ language code.

The compilation of the $\mathrm{C}$ program converts it into machine language file (.hex). This is the only language the microcontroller will understand, because it contains the original program code converted into a hexadecimal format. During this step there are some warnings about eventual errors in the program. If there are no errors and warnings then run the program, the system performs all the required tasks and behaves as expected the software developed. If not, the whole procedure will have to be repeated again.

\section{Results and Discussion}

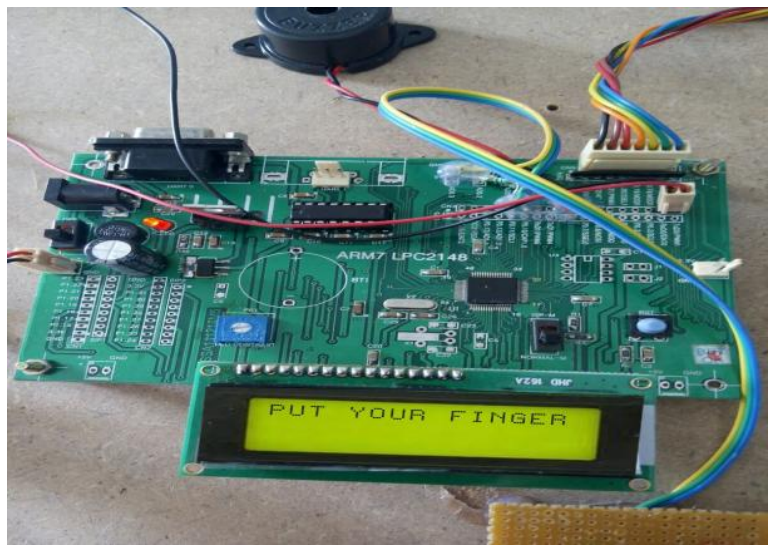

Figure 2: ARM7 Board (LPC2148)
The implementation of realization of "FINGER PRINT BASED AUTHENTICATION SYSTEM USING ARM 7"

is done successfully. The communication is properly done without any interference between different modules in the design. Design is done to meet all the specifications and requirements. Software tools like keil uvision simulator, Flash magic to dump the source code into the microcontroller, orcad lite for the schematic diagram have been used to develop the software code before realizing the hardware.

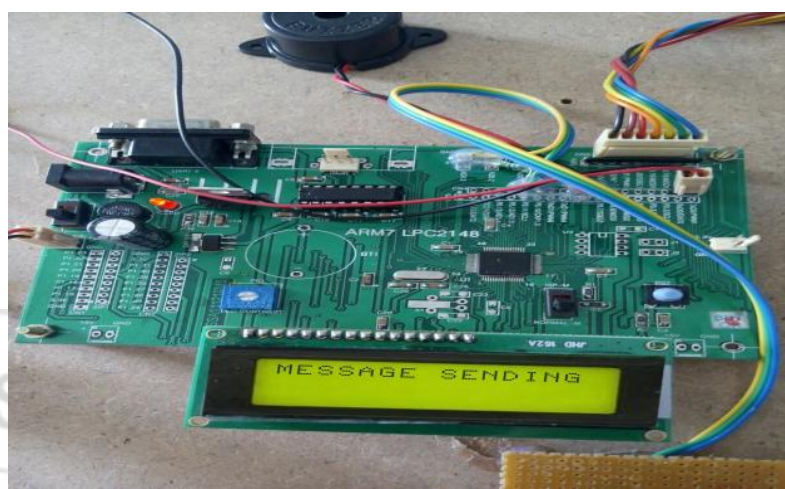

Figure 3: Message sending

Circuit is implemented in Orcad and implemented on the microcontroller board. The performance has been verified both in software simulator and hardware design. The total circuit is completely verified functionally and is following the application software. It can be concluded that the design implemented in the present work provide portability, flexibility and the data transmission is also done with low power consumption.

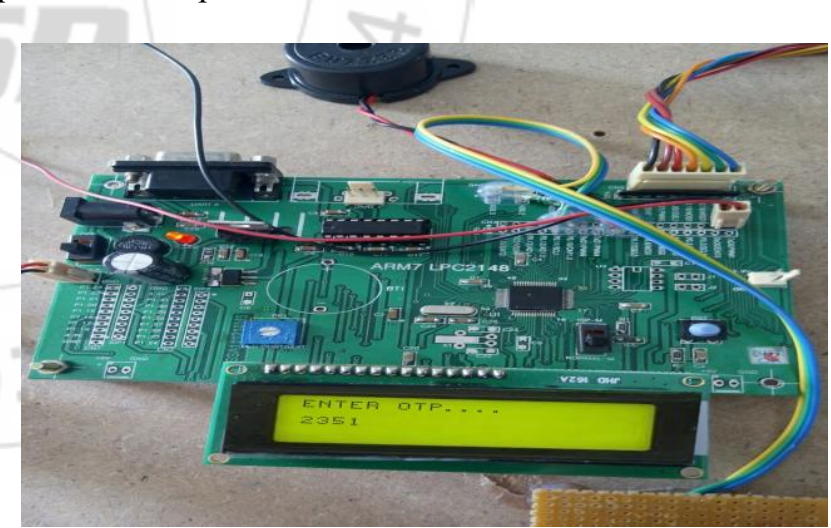

Figure 4: Enter OTP

\section{Conclusion}

We have implemented a locker security system using FINGERPRINT, PASSWORD and GSM. It is a low cost, low in power conception, compact in size and standalone system. The microcontroller compares the passwords entered by keyboard and received through mobile phone. If these passwords are correct the microcontroller provides necessary control signal to open the locker. Future work of this paper is planned to a develop security system based on Iris scanner for visual identification of the person. 


\section{References}

[1] Mary Lourde R and Dushyant Khosla, "Fingerprint Identification in Biometric Security Systems" ,International Journal of Computer and Electrical Engineering, Vol. 2, No. 5, October, 2010.

[2] D. Vinod kumar, Prof.M R K Murthy, “ Fingerprint Based ATM Security by using ARM7”, IOSR Journal of Electronics and Communication Engineering (IOSRJECE) ISSN : 2278-2834.

[3] Hugh Wimberly, Lorie M. Liebrock, "Using Fingerprint Authentication to Reduce SystemSecurity: An Empirical Study", 2011 IEEE Symposium on Security and Privacy.

[4] Kumar Chaturvedula, “ RFID Based Embedded System for Vehicle Tracking and Prevention of Road Accidents", International Journal of Engineering Research \& Technology (IJERT), Vol. 1 Issue 6, August - 2012 .

[5] Gangi.Raghu Ram, N.Rajesh Babu, “ Tracking objects using RFID and Wireless Sensor Networks"ijesat] International Journal Of Engineering science \& Advanced technology Volume-2, Issue-3.

[6] Vishy Karri1, Daniel J.S. Lim2, "Method and Device to Communicate via SMS After a Security Intrusion", 1st International Conference on Sensing Technology November 21-23, 2005 Palmerston North, New Zealand.

[7] Art Conklin1, Glenn Dietrich2, Diane Walz3, "PasswordBased Authentication: A System Perspective", Proceedings of the 37th Hawaii International Conference on System Sciences -2004 .

[8] Zhang Jinhai, Liu Xinjian, Chen Bo, "The design and implementation of ID Authentication System Based on Fingerprint Identification", 2011 Fourth International Conference on Intelligent Computation Technology and Automation.

[9] V.Ramya1, B. Palaniappan, V.Sumathi, "Gsm Based Embedded System For Remote Laboratory Safety Monitoring And Alerting", International Journal of Distributed and Parallel Systems (IJDPS).

\section{Author Profile}

Ambavarapu Bhavana pursuing the M.Tech. Degree in Embedded system of Electronics and communication engineering from Srivenkatesa Perumal College of engineering and technology in 2013-2015 puttur.

M. Jasmine working as an Associate professor in Srivenkatesa Perumal College of engineering and technology in puttur. 